\title{
A Study on Artificial Intelligence
}

\author{
Swaraj Sanas ${ }^{1}$, Srijeet Patil ${ }^{2}$, Om Patil ${ }^{3}$, Prof. Suvarna Nimkarde ${ }^{4}$ \\ Students, Computer Technology, Bharati Vidyapeeth's Institute of Technology, Kharghar, India ${ }^{1,2,3}$ \\ Prof. Computer Technology, Bharati Vidyapeeth's Institute of Technology, Kharghar, India ${ }^{4}$
}

\begin{abstract}
The birth of the field of Artificial Intelligence was primarily due to the curiosity of researchers and inventors to create a perfect replica of the human mind. This research paper aims to provide better understanding of Artificial Intelligence and the terms related to it [7], providing an unique view to the vast technology under our nose left unnoticed by most of the users. Artificial intelligence is used to solve the logical problem ,similar to the human intelligence although the AI can solve the problems even human are not able to because it is not bind by many of the problem human faces. AI solves the problems. It also the subfield of computer science, now a day's becoming popular in power station. AI might be Catalyst [6].
\end{abstract}

Keywords: Artificial Intelligence, Machine Language, Deep Learning

\section{INTRODUCTION}

Artificial Intelligence was found in 1950 by English Mathematician Alan Turing. He published a paper entitled "Computing Machinery and Intelligence" [2]. After this John McCarthy proposed the term "Artificial Intelligence" for it. Artificial Intelligence has ability to provide intelligent behaviour, learn, demonstrate, explain and provide the user best advice. A typical AI observes its environment and takes action that increases its possibility of achieving its goals. Artificial Intelligence is also known as machine Intelligence [3]. The machine Intelligence is opposite to the "natural intelligence". Natural Intelligence is seen in humans as they have evolved for 125,000-65,000 years, Machine Intelligence is more specific compared to the Natural Intelligence. Some people also consider AI to be a danger to humanity if it progresses unsupervised. Others believe that AI will take most of the jobs occupied by human and result in "Mass Unemployment".

\section{LITERATURE REVIEW}

Artificial intelligence is also called as the machine intelligence which is demonstrated by machine and also with natural intelligence showed by humans and other species. In artificial intelligence it is able to design human like Artificial intelligence. But, it is not over, it will take around 50-60 years to come into existence. But, nowadays Robots are made to read, write or to understand the particular task when we see today if we download a simple game like chess and play with the computer at hard stage or any other stage it becomes difficult to win against him. So, this is achieved due to Artificial intelligence [4].Expert system like Google, Yahoo and etc. Suppose we write lst (last) then the search engine will show the possible result of that word like last.

\section{So, this is due to betterment of Artificial intelligence}

Artificial intelligence is a field that covers everything which has anything to do with connecting machines with "intelligence". Artificial intelligence focuses on simulating a human being's distinctive reasoning capability. The goal of $\mathrm{AI}$ is achieved by using complex algorithms and exposing them to the data, and generating an insight which could be useful for future decision making and prediction.

Deep learning: It is a subgroup of machine learning, which is the most advanced field of artificial intelligence, one which brings AI to the proximity of enabling machines to think like humans. Machine Learning: Machine learning is a part of AI, which focuses on providing computer systems access to large volumes of data which helps them to find a way to learn necessary tasks, without having to program them explicitly.

\section{MERITS AND DEMERITS}

\section{Merits}

- Machine learning is used for online advertisement many companies use AI to make their content to the particular user like Google, Facebook and YouTube.

- Best possible solution for a problem such as a theorem, formula can be found with the help of AI, thus AI can provide better and faster result for a problem. 
Vol. 7, Issue 8, August 2018

- It reduces the time cycle (time form beginning to the end of the process) and gives better utilization of the resources.

- It can be used in gaming to provide a unique environment and difficulty to the player giving the player more absorbing game-play.

- $\quad$ Clever chat box can be made using AI [5].

\section{Demerits}

- $\quad$ Artificial Intelligence requires a huge amount of data to be feed to it; such data may not be achieved by smaller organization.

- $\quad$ Due to the huge costs as they are very complex machines artificial technology is costly.

- As machines are able to do the human job it is affordable and convenient to make use of Artificial intelligence rather than hiring an employee this will cause Unemployment.

- They don't improve with experience this is due to how humans and machine stores data which are very different.

\section{APPLICATIONS}

1. Gaming: It is rather more fun to have an opponent with intelligence to play strategic games like chess or a game with bots, Artificial Intelligence provides this experience to the game [1].

2. Visual System: These systems are read, interpret data through visual input. Example: A satellite used for making sense of spatial or maps.

3. Intelligent Robots: Though they cannot replace humans, Robots are able to process; interpret data like light, weight, pressure with help of sensors

4. Handwriting Recognition: This software will read the input given by a pen or stylus and provide an editable text as output.

5. Voice: Machine will understand human language and talk back to them they even understand background noise, slang and accent etc.

\section{METHODOLOGY}

Development of AI was very steady in terms of time there are total three waves of AI development over the years. According to the research the first wave of AI the specialists formulated algorithms and programs based on their knowledge in lacked in ability of learning and way to deal with uncertainty. The second wave of AI was by DARPA they made 15 autonomous vehicles to run a 150 mile track in desert although none of them reached the end point making this whole project a failure. In the last wave of AI the system will take the charge of constructing model themselves this will change the entire decision making and making AI more reliable in the third wave due to the incredible boom of the android and sudden increase in the record breaking number of data source it became the first success full wave of AI.

Flowchart for AI (neural network)

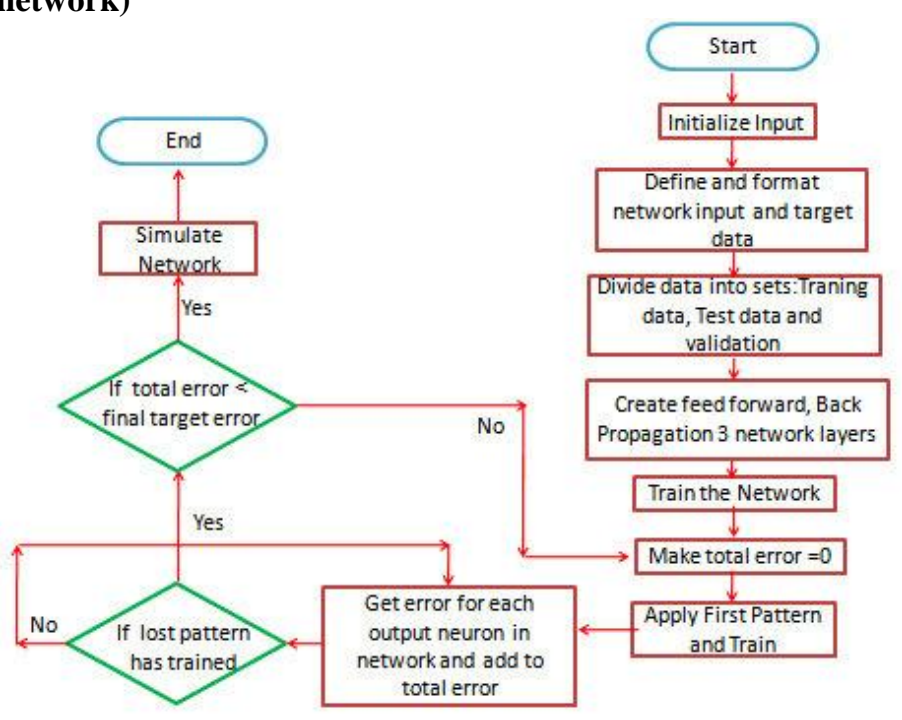




\section{CONCLUSION}

As we move ahead with the world, as AI is increasingly becoming part of our lives and now that it has reached this immensely progressive stage, how far can we go with a system that doesn't know right from wrong? [1]. In conclusion we got a better understanding about Artificial intelligence and the terms related to it. Artificial intelligence is a very powerful technology it can cure the humanity by eradicating the problems such as poverty and hunger although the unintentional ill effects of it cannot be left ignored. Research alone can control the potential harm and allow us to savour the fruit of its innovation.

\section{REFERENCES}

[1]. Rohit Dsouza, Shraddha Ravishankar, Arushi Shah, PurvaRaut, A Model for Ethical Artificial Intelligence, https://www.ijarcce.com/upload 12015/october-15/IJARCCE\%2051.pdf

[2]. D. Saranya ,M.Phil," A Study on Artificial Intelligence and Its Applications",https://www.ijarcce.com/upload/2016/april-16/IJARCCE\% 2080. pdf

[3]. A.Seethai, Dr. Azha. Periasamy, Dr. S. Muruganand, "Rescue Robotics Using Artificial Intelligence", https://ijarcce.com/wp-content/uploads /2012/03/IJARCCE2H-A-seetha-_RESCUE.pdf

[4]. Sparrow, R., "Killer Robots," Journal of Applied Philosophy, 24(1): 62-77, 2007.http://staffwww.dcs.shef.ac.uk/people/A.Sharkey/Sparrow.pdf

[5]. Sarthak V. Doshi, Suprabha B. Pawar, Akshay G. Shelar, Shraddha S. Kulkarni, "Artificial Intelligence Chatbot in Android System using Open Source Program-O", https://ijarcce.com/upload/2017/april-17/IJARCCE\%20151.pdf.

[6]. George Bekey," Autonomous Robots: From Biological Inspiration to Implementation and Control”, MIT Press, Cambridge, MA, 2005. https://www.me.utexas.edu/ longoria/CyVS/notes/references/06_Bekey_Ch5.pdf

[7]. https://data-flair.training/blogs/artificial-intelligence-advantages-disadvantages/

[8]. https://www.newgenapps.com/blog/ai-uses-applications-of-artificial-intelligence-ml-business

[9]. https://futurism.com/1-evergreen-making-sense-of-terms-deep-learning-machine-learning-and-ai/

[10]. https://www.dexlabanalytics.com/blog/learn-to-surf-on-the-three-waves-of-artificial-intelligence 